\title{
Clinical presentation and outcome across age categories among patients with COVID-19 admitted to a Spanish Emergency Department
}

\author{
F. Javier Martín-Sánchez ${ }^{1,2,3}$ (1) Enrique del Toro ${ }^{1} \cdot$ Eduardo Cardassay $^{1} \cdot$ Adrián Valls Carbó $^{4} \cdot$ Federico Cuesta $^{5}$. \\ Marta Vigara $^{5}$. Pedro Gil ${ }^{5}$. Amanda López López Picado ${ }^{3}$. Carmen Martínez Valero ${ }^{3}$. Juande D. Miranda ${ }^{6}$. \\ Pedro Lopez-Ayala ${ }^{7}$. David Chaparro . Gabriel Cozar López $^{1}$ - María del Mar Suárez-Cadenas ${ }^{1,3}$. \\ Pablo Jerez Fernández ${ }^{1}$. Beatriz Angós ${ }^{1}$. Cristina Díaz del Arco $^{8}$ - Esther Rodríguez Adrada ${ }^{3}$. \\ María Teresa Montalvo Moraleda ${ }^{4}$. Carolina Espejo Paeres ${ }^{9} \cdot$ Cesáreo Fernández Alonso $^{1,3} \cdot$ Carlos Elvira $^{10}$. \\ Ana Chacón ${ }^{10}$. Miguel Ángel García Briñón ${ }^{1,3}$. José Luis Fernández Rueda ${ }^{11}$. Luis Ortega ${ }^{8}$. \\ Cristina Fernández Pérez ${ }^{12}$. Juan Jorge González Armengol ${ }^{1,2,3}$. Juan González del Castillo ${ }^{1,3}$
}

Received: 22 May 2020 / Accepted: 8 July 2020 / Published online: 16 July 2020

(c) European Geriatric Medicine Society 2020

\section{Key summary points}

Aim According to age, there are differences in the clinical profile, presentation, management, and short-term outcomes of patients with COVID-19 admitted to the Emergency Department.

Findings A statistically significant association was found between demographic data, comorbidities, clinical, radiographic, analytical, and therapeutic variables and short-term results according to age-dependent categories. Age was a prognostic factor for hospital admission and in-hospital and 30-day mortality, and was associated with not being admitted to intensive care. Message Clinical presentation, management, and short-term outcomes differ according to age among patients with COVID19 admitted to the Emergency Department.

\begin{abstract}
Purpose To determine the differences by age-dependent categories in the clinical profile, presentation, management, and short-term outcomes of patients with laboratory-confirmed COVID-19 admitted to a Spanish Emergency Department (ED). Methods Secondary analysis of COVID-19_URG-HCSC registry. We included all consecutive patients with laboratoryconfirmed COVID-19 admitted to the ED of the University Hospital Clinico San Carlos (Madrid, Spain). The population was divided into six age groups. Demographic, baseline and acute clinical data, and in-hospital and 30-day outcomes were collected.

Results 1379 confirmed COVID-19 cases (mean age 62 (SD 18) years old; $53.5 \%$ male) were included (18.1\%<45 years; $17.8 \%$ 45-54 years; $17.9 \%$ 55-64 years; 17.2\% 65-74 years; $17.0 \%$ 75-84 years; and 11.9\% $\geq 85$ years). A statistically significant association was found between demographic, comorbidity, clinical, radiographic, analytical, and therapeutic variables and short-term results according to age-dependent categories. There were less COVID-specific symptoms and more atypical symptoms among older people. Age was a prognostic factor for hospital admission (aOR = 1.04; 95\% CI 1.02-1.05) and in-hospital (aOR $=1.08 ; 95 \%$ CI 1.05-1.10) and 30-day mortality (aOR =1.07; 95\% CI 1.04-1.09), and was associated with not being admitted to intensive care ( $\mathrm{aOR}=0.95 ; 95 \%$ CI $0.93-0.98)$.
\end{abstract}

F. Javier Martín-Sánchez, Enrique del Toro, and Eduardo

Cardassay have contributed equally to this manuscript and should

be considered first author.

Electronic supplementary material The online version of this article (https://doi.org/10.1007/s41999-020-00359-2) contains supplementary material, which is available to authorized users.

Extended author information available on the last page of the article 
Conclusions Older age is associated with less COVID-specific symptoms and more atypical symptoms, and poor shortterm outcomes. Age has independent prognostic value and may help in shared decision-making in patients with confirmed COVID-19 infection.

Keywords Age $\cdot$ Older $\cdot$ COVID-19 $\cdot$ Emergency department

\section{Introduction}

Since December 2019, when the first case of coronavirus disease-2019 (COVID-19) was reported in Wuhan (Hubei, China), the virus has spread worldwide, causing a global pandemic, which has become an international public health emergency [1]. Spain has been one of the countries most affected in the world, with the region of Madrid presenting some of the highest case and mortality rates [2]. Spain has an increasingly ageing society [3]. A high frequency of deaths by COVID-19 has been documented in older people $[2,4,5]$, with the mortality rate by COVID-19 increasing with age from 5\% in subjects between 60 and 69 years of age to $14.3 \%$ in those between 70 and 79 years and up to $21.3 \%$ in individuals over 80 years old [2].

Older people are characterised by physiological ageing changes, multiple age-related comorbid conditions, and polypharmacy [6]. These circumstances facilitate the presentation of nonspecific and atypical clinical manifestations [4, 7]. The immune system of older adults undergoes several age-related changes, collectively termed immune senescence, involving many cellular and molecular elements of both innate and adaptive immune systems, making this group more vulnerable to infectious diseases [8]. In addition, certain comorbidities such as cardiovascular risk factors, and chronic diseases and cancer have been associated with poor outcomes [9]. All of these factors can contribute to poor health outcomes $[4,7,8]$.

The scientific community is demanding specific information about older people and the establishment of their needs [10]. Unfortunately, the clinical characteristics of COVID-19 infection are little known in this age group. Furthermore, there is not enough evidence about the clinical profile and results in patients admitted to Emergency Departments (EDs). So far, there is little information on age-based clinical features, medical management, and short-term outcomes of COVID-19 in the ED. The objective of the present study was to determine whether there are differences in the clinical profile, presentation, management, and short-term outcomes of patients with laboratory-confirmed COVID-19 admitted to a Spanish ED according to age-dependent categories.

\section{Methods}

\section{Study design}

This is a secondary analysis of the COVID-19_URG-HCSC registry. This registry is a retrospective, observational cohort study, including all suspected COVID-19 cases in the ED of the University Hospital Clinico San Carlos (HCSC).

The HCSC is an urban public tertiary university hospital in Madrid with a reference area of 400,000 inhabitants. The ED of the HCSC attends 140,000 cases per year, with independent Paediatrics, Traumatology, Gynaecology-Obstetrics, and Psychiatry areas. Although a telephone consultation system attended by emergency medical services (EMS) was specifically created during the pandemic and every citizen in our region is assigned a general practitioner (GP), the ED was opened to everyone regardless of the severity of symptoms or previous contact with GPs or the EMS.

The COVID-19_URG-HCSC registry is part of the clinical database that was specifically created for the COVID-19 pandemic (BDCLIN_HCSC_COVID-19). The BDCLIN_ HCSC_COVID-19 is a secure institutional electronic data capture system and repository, which includes the sociodemographic, clinical, diagnostic test, and therapeutic and clinical follow-up data of all the COVID-19 cases handled in the HCSC. Since the 28th of February 2020, the information is being sequentially and additively incorporated from several clinical and administrative sources and will continue to be incorporated throughout the pandemic.

\section{Patient selection}

For the present study, laboratory-confirmed COVID-19 cases admitted to the ED from 28 February 2020 to 31 March 2020 were included. Diagnosis was made by the detection of SARS-CoV-2 RNA by reverse transcription polymerase chain reaction (RT-PCR) in nasopharynx and oropharynx samples. In cases, in which the RT-PCR was repeated, because the result was non-conclusive or a false-negative result was suspected, the last result of the diagnostic test performed during the study period was selected. The study population was divided into six age groups: group 1: 18-44 years old; group 2: 45-54 years old; group 3: 55-64 years old; group 4: 65-74 years old; group 5: 75-84 years old; and group 6: $\geq 85$ years old.

\section{Clinical assessment, data collection and follow-up}

Clinical assessment at the ED included medical history, physical examination, chest radiography, laboratory test, and RT-PCR if available. The criteria for requesting RTPCR were determined by instructions from the Spanish 
Ministry of Health [11], which varied along the study period. Until March 11th, when Madrid was recognized as an area with community transmission, emergency medicine physicians could only order tests for patients admitted for severe viral pneumonia or those who met epidemiological criteria. After this date, SARS-CoV-2 testing was always requested in suspected, more vulnerable, institutionalized, or hospitalized cases. A semi-structured medical record for clinical practice was designed as a modified version of the WHO/International Severe Acute Respiratory and Emerging Infection Consortium Rapid Case Report Form [12] and included clinical symptoms and signs, radiological, laboratory, and COVID-19 treatments. The data of the COVID-19_URG-HCSC registry were entered from medical records by eight investigators using REDCap (Research Electronic Data Capture) (Table in Online Supplementary Material). All the investigators involved in the data collection had been previously trained in information collection. To establish the quality and accuracy of each data included, all the information was checked by a trained researcher. This system ensured the quality of the information included in this study. Patient follow-up was performed 30 days after the index episode by accessing the regional administrative database and hospital and primary care electronic medical records or by telephone contact when necessary to know the vital status of the patients. Patient management was left to the discretion of the attending physician. This guideline was based on recommendations by the Spanish Government and Scientific Societies [13].

\section{Study variables}

Data were collected on sociodemographic (3 variables), medical history (14 variables), chronic treatment (4 variables), baseline functional status (1 variable), clinical symptoms and signs (20 variables), radiological (4 variables), laboratory (17 variables), and COVID-19 treatments (7 variables). The outcomes were hospital admission, high-flow oxygen requirement, intensive-care unit (ICU) admission, and in-hospital and 30-day all-cause mortality.

\section{Statistical analysis}

Continuous variables are presented as mean (standard deviation $[\mathrm{SD}]$ ) or median (interquartile range [IQR]), as appropriate and categorical variables are expressed as numbers and percentages. For the present study, the population was divided into six groups sorted by age-dependent categories. Comparisons among groups were made using the Spearman correlation coefficient for continuous variables and the Chi-square test (or Fisher's exact test when more than
$25 \%$ of the expected frequencies were $<5$ ) was used for categorical variables. $P$ values for linear trend were estimated. Subgroup analysis for clinical and laboratory variables was made. The reference group in the model was the youngest patient group (18-44 years). 30-day cumulative mortality plots stratified by age-dependent groups were plotted using the Kaplan-Meier method, and univariable associations were compared using the log-rank test. Follow-up started at the time of the ED arrival. Outcomes for the different age groups were compared using multivariable logistic regression. Measures of association were expressed as odds ratio (OR), with a 95\% confidence interval (CI), adjusted for sex and co-variables on the basis of clinical interest and relevant prior findings in the literature clinically. Missing values were replaced using the multiple imputation technique, generating five datasets with no missing values for the variables included in the adjustment. Statistically significant differences were considered as a two-side $P$ value less than 0.05 or if the OR 95\% CI excluded 1. The sample size was not calculated as this was an exploratory study. Data analysis was performed using SPSS software, version 24.00 (IBM, New Castle, NY, USA) and STATA version 15.1 (Stata Corp, College Station, TX, USA). Some figures were plotted using R statistical software ( $\mathrm{R}$ foundation for Statistical Computing, Vienna, Austria).

The COVID-19-URG-HCSC registry was developed following the ethical principles of the Declaration of Helsinki for medical research in humans. The protocol and waiver of the need for obtaining informed consent were approved by the HCSC Research Ethics Committee (Cl 20/338-E).

\section{Results}

Out of a total of 2193 patients with suspected COVID-19 admitted to the ED, 1379 laboratory-confirmed cases were included in the analysis, thereby excluding 814 (37.1\%) subjects, because diagnosis had not been confirmed by RT-PCR (363 cases with negative RT-PCR, 19 cases with non-conclusive RT-PCR, and 432 cases in whom RT-PCR was not performed) (Fig. 1). Diagnostic tests were more frequently performed as age increased (Fig. 1 in Online Supplementary Material).

\section{Characteristics of the study subjects}

The median age of the 1379 patients was 63 (IQR $48-77)$ years and $738(53.5 \%)$ were male; $250(18.1 \%)$ were $<45$ years old, $246(17.8 \%) 45-54$ years, $247(17.9 \%)$ 55-64 years, $237(17.2 \%)$ 65-74 years, $235(17.0 \%)$ $75-84$ years, and $164(11.9 \%)$ were 85 years of age or older. The most common comorbidities were hypertension (40.5\%), dyslipidaemia (37.9\%), and diabetes mellitus 


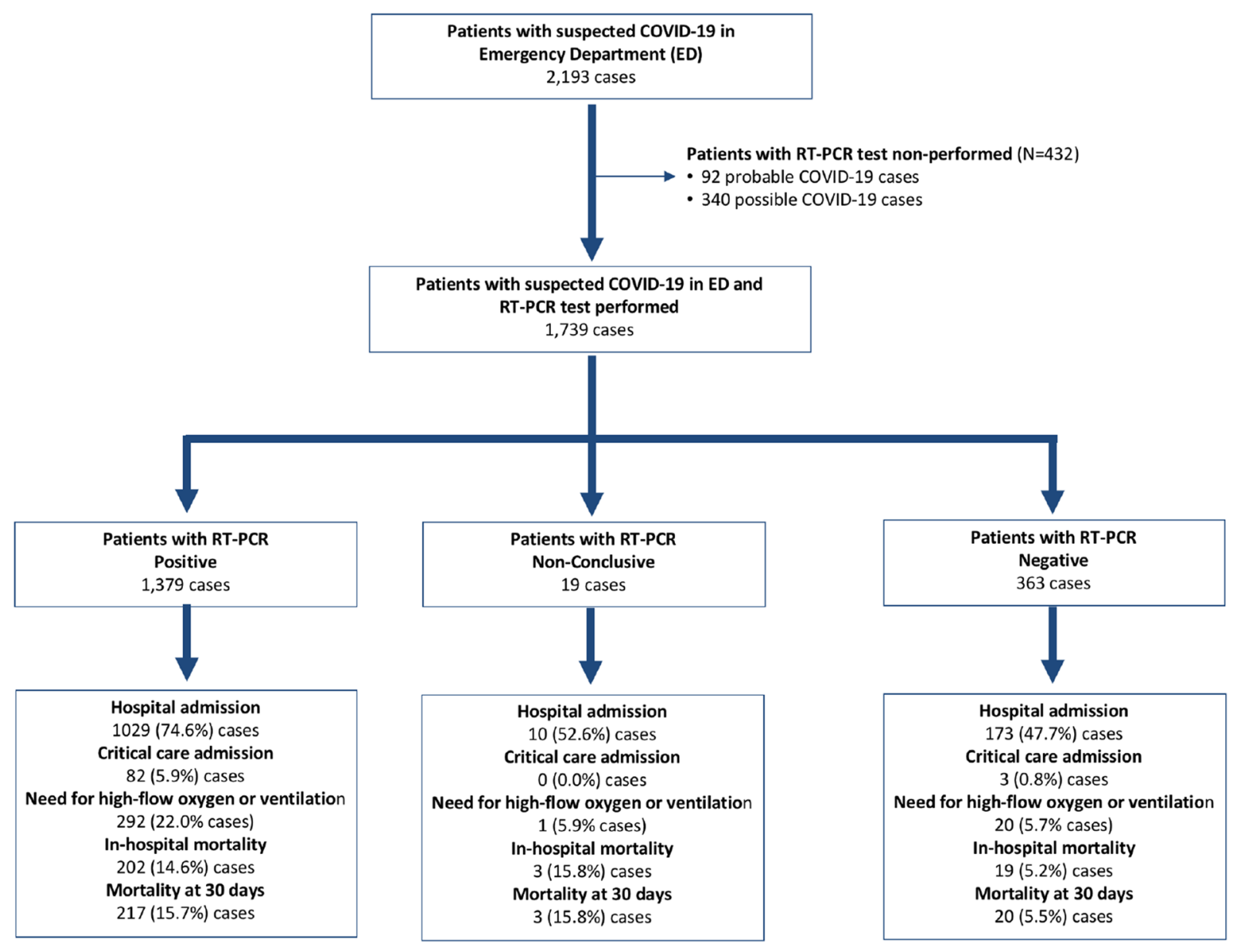

Fig. 1 Flowchart of patient inclusion

(9.2\%). Among the population cohort, $80.0 \%$ had subjective fever, $71.1 \%$ cough, and $47.74 \%$ dyspnoea. Regarding the vital sign at arrival to the ED, $33.7 \%$ had fever $\left(\geq 38{ }^{\circ} \mathrm{C}\right.$ ) and $23.6 \%$ hypoxemia (O2 saturation $<92 \%$ ). Chest X-ray was performed in 1348 patients, $69.8 \%$ of whom showed abnormal results. The most common treatment for COVID-19 was hydroxychloroquine (71.3\%) following by antibiotics (63.7\%), lopinavir/ritonavir (45.6\%), and corticosteroids (19.9\%) (Tables 1, 2 and 3).

\section{Comparison of patient characteristics by age-dependent categories}

Regarding the relationship between cardiovascular risk factors and age groups, there was a higher frequency of hypertension $(P<0.001)$, dyslipidaemia $(P<0.001)$, and diabetes mellitus $(P<0.001)$ with increasing age. Conversely, there was a lower percentage of current smokers $(P<0.001)$ (Table 1).
With respect to comorbidities, there was an increase in cancer and all degenerative and chronic diseases with age, except for HIV. Simultaneously, there was a higher frequency of treatments with angiotensin-converting enzyme inhibitors (ACEs)/angiotensin II receptor blockers (ARBs) $(P<0.001)$, anticoagulation $(P<0.001)$, antiplatelet $(P<0.001)$, and immunosuppressive agents $(P<0.001)$ with increasing age (Table 1).

Statistically significant differences by age-dependent categories were also found in terms of clinical symptoms and signs. Older subjects presented fewer core COVID-19 symptoms and more typical geriatric complaints. The population aged 65 and older showed a higher percentage of patients with confusion $(P<0.001)$, asthenia $(P=0.033)$, and pre/ syncope $(P<0.001)$ (Table 2$)$.

There was an indirect relationship between age and oxygen saturation $(P<0.001)$ and body temperature $(P=0.002)$ and a direct relationship between age and systolic blood pressure $(P=0.043)$ at arrival to the ED (Table 2). 
Table 1 Socio-demographic and medical history data of the study population by age-dependent categories

\begin{tabular}{|c|c|c|c|c|c|c|c|c|}
\hline & $\begin{array}{l}\text { Patients } \\
N=1379 \\
N(\%)\end{array}$ & $\begin{array}{l}18-44 \text { years, } \\
N=250 \\
n(\%)\end{array}$ & $\begin{array}{l}45-54 \text { years, } \\
N=246 \\
n(\%)\end{array}$ & $\begin{array}{l}55-64 \text { years, } \\
N=247 \\
n(\%)\end{array}$ & $\begin{array}{l}65-74 \text { years, } \\
N=237 \\
n(\%)\end{array}$ & $\begin{array}{l}75-84 \text { years, } \\
N=235 \\
n(\%)\end{array}$ & $\begin{array}{l}\geq 85 \text { years, } \\
N=164 \\
n(\%)\end{array}$ & $P$ value \\
\hline \multicolumn{9}{|c|}{ Socio-demographic data } \\
\hline Male gender & $739(53.6)$ & $136(54.4)$ & $133(54.1)$ & $124(50.2)$ & $140(59.1)$ & $125(53.2)$ & $80(48.8)$ & 0.545 \\
\hline $\begin{array}{l}\text { Living in } \\
\text { nursing } \\
\text { home }\end{array}$ & $33(2.4)$ & $1(0.4)$ & $2(0.8)$ & $1(0.4)$ & $4(1.7)$ & $6(2.6)$ & $19(11.7)$ & $<0.001$ \\
\hline \multicolumn{9}{|l|}{ Medical history } \\
\hline $\begin{array}{l}\text { Current smok- } \\
\text { ing }\end{array}$ & $61(4.4)$ & $9(3.6)$ & $9(3.7)$ & $13(5.3)$ & $21(8.9)$ & $6(2.6)$ & $3(1.8)$ & $<0.001$ \\
\hline Hypertension & $559(40.5)$ & $6(2.4)$ & $41(16.7)$ & 78 (31.6) & $148(62.4)$ & $167(71.1)$ & $119(72.6)$ & $<0.001$ \\
\hline $\begin{array}{l}\text { Diabetes mel- } \\
\text { litus }\end{array}$ & $265(19.2)$ & $7(2.8)$ & $17(6.9)$ & $35(14.2)$ & $73(30.8)$ & $80(34.0)$ & $53(32.3)$ & $<0.001$ \\
\hline Dyslipidemia & $523(37.9)$ & $8(3.2)$ & $38(15.4)$ & $96(38.9)$ & $146(61.6)$ & $147(62.6)$ & $88(53.7)$ & $<0.001$ \\
\hline Obesity & $131(9.5)$ & $20(8.0)$ & $25(10.2)$ & $26(10.5)$ & $28(11.8)$ & $24(10.2)$ & $8(4.9)$ & 0.664 \\
\hline $\begin{array}{l}\text { Chronic } \\
\text { respiratory } \\
\text { disease }\end{array}$ & $239(17.3)$ & $17(6.8)$ & $24(9.8)$ & $30(12.1)$ & $59(24.9)$ & $62(26.4)$ & $47(28.7)$ & $<0.001$ \\
\hline Heart disease & $237(17.2)$ & $6(2.4)$ & $7(2.8)$ & $19(7.7)$ & 49 (20.7) & 70 (29.8) & $86(52.4)$ & $<0.001$ \\
\hline $\begin{array}{l}\text { Peripheral } \\
\text { artery dis- } \\
\text { ease }\end{array}$ & $36(2.6)$ & $0(0.0)$ & $0(0.0)$ & $3(1.2)$ & $7(3.0)$ & $13(5.5)$ & $13(7.9)$ & $<0.001$ \\
\hline $\begin{array}{l}\text { Cerebrovascu- } \\
\text { lar disease }\end{array}$ & $58(4.2)$ & $0(0.0)$ & $1(0.4)$ & $3(1.2)$ & $15(6.3)$ & $24(10.2)$ & $15(9.1)$ & $<0.001$ \\
\hline Dementia & $67(4.9)$ & $0(0.0)$ & $1(0.4)$ & $1(0.4)$ & $8(3.4)$ & $18(7.7)$ & $39(23.8)$ & $<0.001$ \\
\hline $\begin{array}{l}\text { Chronic renal } \\
\text { disease }\end{array}$ & $109(7.9)$ & $1(0.4)$ & $7(2.8)$ & $11(4.5)$ & $29(12.2)$ & $39(16.6)$ & $22(13.4)$ & $<0.001$ \\
\hline Liver disease & $43(3.1)$ & $0(0.0)$ & $8(3.3)$ & $11(4.5)$ & $10(4.2)$ & $7(3.0)$ & $7(4.3)$ & $\mathbf{0 . 0 3 1}$ \\
\hline Cancer & $130(9.4)$ & $4(1.6)$ & $13(5.3)$ & $19(7.7)$ & $30(12.7)$ & $41(17.4)$ & $23(14.0)$ & $<0.001$ \\
\hline HIV & $16(1.2)$ & $4(1.6)$ & $5(2.0)$ & $4(1.6)$ & $3(1.3)$ & $0(0.0)$ & $0(0.0)$ & $\mathbf{0 . 0 2 0}$ \\
\hline $\begin{array}{l}\text { Connec- } \\
\text { tive tissue } \\
\text { disease }\end{array}$ & $20(1.4)$ & $0(0.0)$ & $5(2.0)$ & $3(1.2)$ & $3(0.8)$ & $4(1.7)$ & $6(3.7)$ & 0.012 \\
\hline \multicolumn{9}{|c|}{ Baseline functional status } \\
\hline $\begin{array}{l}\text { Independent } \\
\text { BADL }\end{array}$ & $1220(91.4)$ & $246(98.8)$ & $235(98.3)$ & 237 (97.9) & $217(94.3)$ & $198(87.2)$ & $87(58.8)$ & $<0.001$ \\
\hline $\begin{array}{l}\text { Partially } \\
\text { dependent } \\
\text { BADL }\end{array}$ & $64(4.8)$ & $0(0.0)$ & $2(0.8)$ & $5(2.1)$ & $8(3.5)$ & $17(7.5)$ & $32(21.6)$ & \\
\hline $\begin{array}{l}\text { Total depend- } \\
\text { ent BADL }\end{array}$ & $51(3.8)$ & $3(1.2)$ & $2(0.8)$ & $0(0.0)$ & $5(2.2)$ & $12(5.3)$ & $29(19.6)$ & \\
\hline \multicolumn{9}{|c|}{ Chronic treatment at home } \\
\hline $\begin{array}{r}\text { ACEis / } \\
\text { ARBs }\end{array}$ & 440 (31.9) & $6(2.4)$ & $30(12.2)$ & $63(25.5)$ & $111(46.8)$ & $138(58.7)$ & $92(56.1)$ & $<0.001$ \\
\hline $\begin{array}{l}\text { Anticoagula- } \\
\text { tion }\end{array}$ & $124(9.0)$ & $1(0.4)$ & $2(0.8)$ & $9(3.6)$ & $20(8.4)$ & $43(18.3)$ & $49(29.9)$ & $<0.001$ \\
\hline $\begin{array}{l}\text { Antiplatelet } \\
\text { agent }\end{array}$ & $208(15.1)$ & $1(0.4)$ & $10(4.1)$ & $20(8.1)$ & $52(21.9)$ & $66(28.1)$ & $59(36.0)$ & $<0.001$ \\
\hline $\begin{array}{l}\text { Immunosup- } \\
\text { pressive } \\
\text { treatment }\end{array}$ & $95(6.9)$ & $3(1.2)$ & $13(5.3)$ & $16(6.5)$ & $22(9.3)$ & $24(10.2)$ & $17(10.4)$ & $<0.001$ \\
\hline
\end{tabular}

$P$ value for lineal trend. Numbers in bold mean value $P<0.05$

$H I V$ human immunodeficiency virus, $B A D L$ basic activities of daily living, $A C E$ angiotensin-converting enzyme inhibitors, ARBs angiotensin II receptor blockers 
Table 2 Clinical data at presentation of the study population by age-dependent categories

\begin{tabular}{|c|c|c|c|c|c|c|c|c|}
\hline & $\begin{array}{l}\text { Patients } \\
N=1379 \\
n(\%)\end{array}$ & $\begin{array}{l}18-44 \text { years, } N \\
=250 \\
n(\%)\end{array}$ & $\begin{array}{l}45-54 \text { years, } \\
N=246 \\
n(\%)\end{array}$ & $\begin{array}{l}55-64 \text { years, } \\
N=247 \\
n(\%)\end{array}$ & $\begin{array}{l}65-74 \text { years, } \\
N=237 \\
n(\%)\end{array}$ & $\begin{array}{l}75-84 \text { years, } \\
N=235 \\
n(\%)\end{array}$ & $\begin{array}{l}>=85 \text { years, } \\
N=164 \\
n(\%)\end{array}$ & $P$ value \\
\hline \multicolumn{9}{|c|}{ Acute episode clinical data } \\
\hline $\begin{array}{l}\text { Time of evo- } \\
\text { lution, days } \\
\text { [median } \\
\text { (IQR)] }\end{array}$ & $7(3-9)$ & $3(6-7)$ & $4(7-9)$ & $4(7-9)$ & $3(7-10)$ & $4(7-8)$ & $2.5(4-7)$ & 0.657 \\
\hline Cough & $981(71.1)$ & $196(78.1)$ & $170(69.1)$ & $189(76.5)$ & $168(70.9)$ & $158(67.2)$ & $100(61.0)$ & $<0.001$ \\
\hline Fever & $1104(80.0)$ & $208(83.2)$ & $204(82.9)$ & $207(83.8)$ & $193(81.4)$ & $180(76.6)$ & $111(67.7)$ & $<0.001$ \\
\hline Dyspnoea & $653(47.3)$ & $120(47.8)$ & $121(49.2)$ & $123(49.8)$ & $115(48.5)$ & $100(42.6)$ & $74(45.1)$ & 0.222 \\
\hline Chest pain & $158(11.4)$ & $38(15.1)$ & $36(14.6)$ & $34(13.8)$ & $31(13.1)$ & $11(4.7)$ & $8(4.9)$ & $<0.001$ \\
\hline Diarrhoea & 275 (19.9) & $42(16.8)$ & $56(22.8)$ & $62(25.1)$ & $59(24.9)$ & $39(16.6)$ & $16(9.8)$ & 0.056 \\
\hline $\begin{array}{c}\text { Nausea and } \\
\text { vomiting }\end{array}$ & $137(9.9)$ & $35(13.9)$ & $22(8.9)$ & $27(10.9)$ & $30(12.7)$ & $18(7.7)$ & $5(3.0)$ & 0.002 \\
\hline Headache & $148(10.7)$ & $41(16.3)$ & $41(16.7)$ & $32(13.0)$ & $17(7.2)$ & $9(3.8)$ & $8(4.9)$ & $<0.001$ \\
\hline Confusion & $38(2.8)$ & $0(0.0)$ & $1(0.4)$ & $1(0.4)$ & $8(3.4)$ & $12(5.1)$ & $16(9.8)$ & $<0.001$ \\
\hline Anosmia & $57(4.1)$ & $15(6.0)$ & $23(9.3)$ & $10(4.0)$ & $8(3.4)$ & $0(0.0)$ & $1(0.6)$ & $<0.001$ \\
\hline Dysgeusia & $59(4.3)$ & $12(4.8)$ & $18(7.3)$ & $15(6.1)$ & $11(4.6)$ & $2(0.9)$ & $1(0.6)$ & $<0.001$ \\
\hline Myalgia & 313 (22.7) & $73(29.2)$ & $72(29.3)$ & $74(30.0)$ & $40(16.9)$ & $35(14.9)$ & $18(11.0)$ & $<0.001$ \\
\hline Asthenia & $449(32.5)$ & $63(25.2)$ & $79(32.1)$ & $82(33.2)$ & $90(38.0)$ & $82(34.9)$ & $53(32.3)$ & $\mathbf{0 . 0 3 3}$ \\
\hline Sore throat & $89(6.4)$ & $27(10.8)$ & $19(7.7)$ & $18(7.3)$ & $9(3.8)$ & $12(5.1)$ & $4(2.4)$ & $<0.001$ \\
\hline Rhinorrhoea & $27(2.0)$ & $12(4.8)$ & $7(2.8)$ & $4(1.6)$ & $0(0.0)$ & $1(0.4)$ & $3(1.8)$ & 0.001 \\
\hline Skin lesions & $4(0.3)$ & $2(0.8)$ & $0(0.0)$ & $0(0.0)$ & $1(0.4)$ & $1(0.4)$ & $0(0.0)$ & 0.484 \\
\hline $\begin{array}{l}\text { Presyncope or } \\
\text { syncope }\end{array}$ & $68(4.9)$ & $0(0.0)$ & $11(4.5)$ & $7(2.8)$ & $14(5.9)$ & $20(8.5)$ & $16(9.8)$ & $<0.001$ \\
\hline \multicolumn{9}{|l|}{ Physical data } \\
\hline $\begin{array}{l}\text { Oxygen } \\
\text { saturation, } \\
\% \text { [mean } \\
\text { (SD)] }\end{array}$ & $94(6.6)$ & $97(3.1)$ & $96(5.7)$ & $94(6.2)$ & $93(6.5)$ & $91(7.8)$ & $91(7.4)$ & $<0.001$ \\
\hline $\begin{array}{l}\text { Body tem- } \\
\text { perature, }{ }^{\circ} \mathrm{C} \\
{[\text { mean }(\mathrm{SD})]}\end{array}$ & $37.5(2.9)$ & $37.7(4.2)$ & $37.4(1.0)$ & $37.7(4.0)$ & $37.3(0.9)$ & $37.5(3.4)$ & $37.1(0.7)$ & 0.002 \\
\hline $\begin{array}{l}\mathrm{SBP}, \mathrm{mmHg} \\
{[\text { mean (SD)] }}\end{array}$ & $130(20.8)$ & $125(17.8)$ & $129(18.2)$ & $129(19.1)$ & $132(19.9)$ & $128(22.1)$ & $134(24.8)$ & 0.043 \\
\hline
\end{tabular}

$P$ value for lineal trend. Numbers in bold mean value $P<0.05$

$S B P$ systolic blood pressure, $I Q R$ interquartile range, $S D$ standard deviation

Although there was no significant difference between age groups according to time of symptom evolution, radiological and laboratory findings related to a poor prognosis were more common with increasing age. This age association was clinically relevant in relation to lymphopenia $(P<0.001)$, thrombocytopenia $(P<0.001)$, a reduction in the glomerular filtration rate $(P<0.001)$, and an increase of lactate dehydrogenase $(P=0.010)$ and $\mathrm{D}$-dimer values $(P<0.001)$ as well as $C$-reactive protein $(P<0.001)$ and procalcitonin $(P<0.001)$ (Table 3$)$.

Concerning immediate treatment, there was greater use of hydroxychloroquine $(P<0.001)$, lopinavir/ritonavir $(\mathrm{P}=0.002)$, corticosteroids $(P<0.001)$, azithromycin $(P<0.001)$, and other antibiotics $(P<0.001)$ with increasing age, with a sharp decline in the use of treatment for COVID-19 in the group $\geq 85$ years of age (Table 3 ).

\section{In-hospital and 30-day outcomes according to age-dependent categories}

Among the 1379 patients, 1030 (74.6\%) were hospitalized (5.9\% in the ICU), and of these, $292(21.9 \%)$ received high-flow oxygen or ventilation and 202 (14.6\%) died during hospitalisation. During the 30-day follow-up, 217 (15.7\%) patients died. A statistically significant linear trend was found between age-dependent groups and the likelihood of hospitalisation, and in-hospital and 30-day mortality (Table 3). 
Table 3 Radiographic, laboratory, and treatment data and outcomes of the study population by age-dependent categories

\begin{tabular}{|c|c|c|c|c|c|c|c|c|}
\hline & $\begin{array}{l}\text { Patients } \\
N=1379 \\
N(\%)\end{array}$ & $\begin{array}{l}18-44 \text { years, } \\
N=250 \\
n(\%)\end{array}$ & $\begin{array}{l}45-54 \text { years, } \\
N=246 \\
n(\%)\end{array}$ & $\begin{array}{l}55-64 \text { years, } \\
N=247 \\
n(\%)\end{array}$ & $\begin{array}{l}65-74 \text { years, } \\
N=237 \\
n(\%)\end{array}$ & $\begin{array}{l}75-84 \text { years, } \\
N=235 \\
n(\%)\end{array}$ & $\begin{array}{l}\geq 85 \text { years, } \\
N=164 \\
n(\%)\end{array}$ & $P$ value \\
\hline \multicolumn{9}{|l|}{ Chest X-ray } \\
\hline $\begin{array}{l}\text { No consolida- } \\
\text { tion }\end{array}$ & 407 (29.5) & $100(40.0)$ & 78 (31.7) & $70(28.3)$ & $57(24.1)$ & $62(26.4)$ & $40(24.4)$ & \multirow[t]{4}{*}{0.002} \\
\hline $\begin{array}{l}\text { Unilateral } \\
\text { consolida- } \\
\text { tion }\end{array}$ & $311(22.5)$ & $57(22.8)$ & $55(22.4)$ & 48 (19.4) & $60(25.3)$ & 44 (18.7) & $46(28.0)$ & \\
\hline $\begin{array}{l}\text { Bilateral con- } \\
\text { solidation }\end{array}$ & $631(45.7)$ & $80(32.0)$ & 103 (41.9) & $126(51.0)$ & $118(49.8)$ & $127(54.0)$ & $77(47.0)$ & \\
\hline Not done & $31(2.2)$ & $13(5.2)$ & $10(4.1)$ & $3(1.2)$ & $2(0.8)$ & $2(0.9)$ & $1(0.6)$ & \\
\hline \multicolumn{9}{|l|}{ Laboratory data } \\
\hline $\begin{array}{l}\text { Leuko- } \\
\text { cytes } \times 10^{3} / \\
\mu \mathrm{L},[\text { median } \\
\text { (IQR)] }\end{array}$ & $4.7(6.1-8.3)$ & $4.8(6.0-7.8)$ & $4.5(6.1-7.6)$ & $4.8(6.4-8.7)$ & $4.5(5.4-8.1)$ & $4.8(6.3-8.5)$ & $4.6(6.4-9.1)$ & 0.267 \\
\hline $\begin{array}{l}\text { Hematocrit, } \\
\% \text { [median } \\
\text { (IQR)] }\end{array}$ & $42.0(4.9)$ & $43.4(4.3)$ & $43.3(4.0)$ & $42.8(4.2)$ & $41.6(5.0)$ & $40.9(5.0)$ & $39.5(5.8)$ & $<0.001$ \\
\hline $\begin{array}{l}\text { Lympho- } \\
\text { cytes } \times 10^{3} / \\
\mu \mathrm{L}[\mathrm{median} \\
(\mathrm{IQR})]\end{array}$ & $0.6(0.9-1.3)$ & $0.8(1.2-1.6)$ & $0.8(1.1-1.4)$ & $0.7(1.0-1.4)$ & $0.6(0.8-1.1)$ & $0.5(0.8-1.1)$ & $0.5(0.7-1.1)$ & $<0.001$ \\
\hline $\begin{array}{l}\text { Neutro- } \\
\text { phils } \times 10^{3} / \\
\mu L[\text { median } \\
\text { (IQR)] }\end{array}$ & $3.2(4.4-6.6)$ & $3.2(4.2-5.6)$ & $3.1(4.3-5.8)$ & $3.3(4.5-7.0)$ & $3.0(4.1-6.6)$ & $3.4(4.7-6.6)$ & $3.3(4.7-7.4)$ & 0.003 \\
\hline $\begin{array}{l}\text { Platelet } \\
\text { count } \times 10^{3} / \\
\mu \mathrm{L}[\text { mean } \\
\text { (SD)] }\end{array}$ & 206.7 (88.6) & $228.1(77.8)$ & $222.2(97.2)$ & $219.3(93.4)$ & $194.2(85.9)$ & $190.9(87.8)$ & $137(224.5)$ & $<0.001$ \\
\hline $\begin{array}{l}\text { GFR }(\mathrm{mL} / \\
\mathrm{min} / 1,73 \\
\mathrm{~m} 2)[\mathrm{mean} \\
\text { (SD)] }\end{array}$ & $79.3(27.9)$ & $109.6(15.1)$ & $94.2(20.2)$ & $84.7(22.2)$ & $71.3(23.5)$ & $62.3(23.7)$ & $55.4(21.7)$ & $<0.001$ \\
\hline $\begin{array}{l}\text { LDH, U/L } \\
\text { [median } \\
\text { (IQR)] }\end{array}$ & $484(624-825)$ & $448(565-708)$ & $486(607-806)$ & $504(647-844)$ & $487(638-867)$ & 507 (656-874) & $460(621-842)$ & 0.010 \\
\hline $\begin{array}{l}\text { D-Dimer, ng/ } \\
\text { ml [median } \\
\text { (IQR)] }\end{array}$ & $\begin{array}{l}394(657- \\
1089)\end{array}$ & $293(454-662)$ & $340(513-778)$ & $360(580-926)$ & $\begin{array}{c}476(783- \\
1380)\end{array}$ & $\begin{array}{l}613(912- \\
1775)\end{array}$ & $\begin{array}{l}652(1140- \\
1974)\end{array}$ & $<0.001$ \\
\hline $\begin{array}{l}\text { C-reactive } \\
\text { protein, mg/ } \\
\text { dl [median } \\
\text { (IQR)] }\end{array}$ & $2.1(5.9-12.3)$ & $1.4(4.0-8.9)$ & $1.4(3.5-9.5)$ & $1.7(6.5-11.6)$ & $2.8(6.3-14.8)$ & $3.3(8.1-14.1)$ & $3.7(8.1-13.7)$ & $<0.001$ \\
\hline $\begin{array}{l}\text { Procalci- } \\
\text { tonin, ng/ } \\
\text { ml [median } \\
\text { (IQR)] }\end{array}$ & $0.0(0.1-0.2)$ & $0.0(0.1-0.1)$ & $0.0(0.1-0.1)$ & $0.0(0.1-0.2)$ & $0.0(0.1-0.2)$ & $0.1(0.1-0.3)$ & $0.1(0.1-0.2)$ & $<0.001$ \\
\hline $\begin{array}{l}\text { Ferritin, ng/ } \\
\text { ml [median } \\
\text { (IQR)] }\end{array}$ & $195(490-958)$ & $114(378-736)$ & $178(461-849)$ & $\begin{array}{l}253(532- \\
1014)\end{array}$ & $\begin{array}{c}335(736- \\
1118)\end{array}$ & $\begin{array}{c}210(487- \\
1139)\end{array}$ & $202(335-722)$ & 0.003 \\
\hline $\begin{array}{c}\text { AST, U/L } \\
\text { [median } \\
\text { (IQR)] }\end{array}$ & $28(38-55)$ & $28(36-55)$ & $28(38-58)$ & $29(39-60)$ & $30(40-60)$ & $27(38-53)$ & $25(34-45)$ & 0.127 \\
\hline
\end{tabular}


Table 3 (continued)

\begin{tabular}{|c|c|c|c|c|c|c|c|c|}
\hline & $\begin{array}{l}\text { Patients } \\
N=1379 \\
N(\%)\end{array}$ & $\begin{array}{l}18-44 \text { years, } \\
N=250 \\
n(\%)\end{array}$ & $\begin{array}{l}45-54 \text { years, } \\
N=246 \\
n(\%)\end{array}$ & $\begin{array}{l}55-64 \text { years, } \\
N=247 \\
n(\%)\end{array}$ & $\begin{array}{l}65-74 \text { years, } \\
N=237 \\
n(\%)\end{array}$ & $\begin{array}{l}75-84 \text { years, } \\
N=235 \\
n(\%)\end{array}$ & $\begin{array}{l}\geq 85 \text { years, } \\
N=164 \\
n(\%)\end{array}$ & $P$ value \\
\hline $\begin{array}{c}\text { ALT, U/L } \\
\text { [median } \\
\text { (IQR)] }\end{array}$ & $18(28-46)$ & $23(34-60)$ & $24(35-58)$ & $20(30-52)$ & $19(29-46)$ & $17(24-36)$ & $14(17-27)$ & $<0.001$ \\
\hline $\begin{array}{l}\text { Bilirrubin, } \\
\text { mg/dL } \\
\text { [median } \\
\text { (IQR)] }\end{array}$ & $0.4(0.5-0.7)$ & $0.4(0.4-0.6)$ & $0.4(0.6-0.7)$ & $0.4(0.5-0.7)$ & $0.4(0.6-0.7)$ & $0.4(0.6-0.8)$ & $0.4(0.6-0.7)$ & $<0.001$ \\
\hline $\begin{array}{l}\text { APTT ratio, } \\
\text { [median } \\
\text { (IQR)] }\end{array}$ & $0.9(1.0-1.1)$ & $1.0(1.0-1.1)$ & $0.9(1.0-1.1)$ & $0.9(1.0-1.1)$ & $0.9(1.0-1.1)$ & $0.9(1.0-1.1)$ & $0.9(1.0-1.1)$ & 0.006 \\
\hline $\begin{array}{l}\text { INR, [median } \\
\text { (IQR)] }\end{array}$ & $1.0(1.1-1.2)$ & $1.0(1.1-1.2)$ & $1.0(1.1-1.2)$ & $1.0(1.1-1.2)$ & $1.0(1.1-1.2)$ & $1.0(1.1-1.3)$ & $1.0(1.1-1.5)$ & $<0.001$ \\
\hline $\begin{array}{l}\text { Fibrinogen, } \\
\text { mg/dl } \\
\text { [median } \\
\text { (IQR)] }\end{array}$ & $603(714-836)$ & $588(718-810)$ & $573(705-833)$ & $616(748-892)$ & $653(746-846)$ & $612(713-823)$ & $569(656-727)$ & 0.660 \\
\hline \multicolumn{9}{|c|}{ Treatment for COVID-19 } \\
\hline $\begin{array}{l}\text { Hydroxychlo- } \\
\text { roquine }\end{array}$ & $979(71.3)$ & 129 (51.6) & $162(66.7)$ & $186(75.6)$ & $201(85.2)$ & $195(83.3)$ & $105(64.0)$ & $<0.001$ \\
\hline $\begin{array}{c}\text { Lopinavir/ } \\
\text { ritonavir }\end{array}$ & $627(45.6)$ & $105(42.0)$ & $119(49.0)$ & $135(54.9)$ & $130(55.1)$ & $98(41.9)$ & $39(23.8)$ & 0.002 \\
\hline Remdesivir & $2(0.1)$ & $0(0.0)$ & $0(0.0)$ & $0(0.0)$ & $1(0.4)$ & $0(0.0)$ & $1(0.6)$ & 0.152 \\
\hline $\begin{array}{l}\text { Intravenous } \\
\text { corticoster- } \\
\text { oid }\end{array}$ & 273 (19.9) & $16(6.4)$ & $27(11.1)$ & $67(27.2)$ & $69(29.2)$ & $64(27.4)$ & $30(18.3)$ & $<0.001$ \\
\hline Tocilizumab & $74(5.4)$ & $3(1.2)$ & $16(6.6)$ & $32(13.0)$ & $15(6.4)$ & $8(3.4)$ & $0(0.0)$ & 0.269 \\
\hline Azithromycin & $277(20.2)$ & $30(12.0)$ & $42(17.3)$ & 39 (15.9) & $65(27.5)$ & $61(26.1)$ & $40(24.4)$ & $<0.001$ \\
\hline $\begin{array}{l}\text { Another } \\
\text { systemic } \\
\text { antibiotic }\end{array}$ & $598(43.5)$ & $52(20.8)$ & $70(28.8)$ & 97 (39.4) & $133(56.4)$ & $145(62.0)$ & $100(61.0)$ & $<0.001$ \\
\hline \multicolumn{9}{|l|}{ Outcomes } \\
\hline $\begin{array}{l}\text { Hospital } \\
\text { admission }\end{array}$ & $1029(74.6)$ & $125(50.0)$ & $151(61.4)$ & 177 (71.7) & $204(86.1)$ & 216 (91.9) & $156(95.1)$ & $<0.001$ \\
\hline $\begin{array}{c}\text { Critical care } \\
\text { admission }\end{array}$ & $82(5.9)$ & $8(3.2)$ & $15(6.1)$ & $30(12.1)$ & $21(8.9)$ & $8(3.4)$ & $0(0.0)$ & 0.151 \\
\hline $\begin{array}{l}\text { Need to high- } \\
\text { flow oxygen } \\
\text { or ventila- } \\
\text { tion }\end{array}$ & $292(22.0)$ & $10(4.1)$ & $25(10.5)$ & $56(23.7)$ & $64(28.1)$ & $86(37.9)$ & $51(32.5)$ & $<0.001$ \\
\hline $\begin{array}{r}\text { In-hospital } \\
\text { mortality }\end{array}$ & 202 (14.6) & $0(0.0)$ & $4(1.6)$ & $10(4.0)$ & $38(16.0)$ & $76(32.3)$ & $74(45.1)$ & $<0.001$ \\
\hline $\begin{array}{l}\text { 30-day mor- } \\
\text { tality }\end{array}$ & $217(15.7)$ & $1(0.4)$ & $6(2.4)$ & $13(5.3)$ & $40(16.9)$ & 79 (33.6) & 78 (47.6) & $<0.001$ \\
\hline
\end{tabular}

$P$ value for lineal trend. Numbers in bold mean value $P<0.05$

GFR glomerular filtration rate, $L D H$ lactate dehydrogenase, $A S T$ aspartate aminotransferase, $A L T$ alanine aminotransferase, $S D$ standard deviation, $I Q R$ interquartile range

Kaplan-Meier survival curves showed statistically significant differences between each age group and the 30-day mortality outcome $(P<0.001)$ (Fig. 2$)$. The oldest age groups were associated with the poorest outcomes.
The forest plot reflects the univariable and multivariable associations between each age, as a continuous variable, and the primary and secondary outcomes. After adjustment for potential confounding factors, age remained significantly associated with hospital admission and in-hospital and 
30-day mortality and was associated with not being admitted to intensive care (Fig. 3).

\section{Discussion}

The main finding of this study is that age determines the profile, management, and short-term health outcomes among COVID-19 patients admitted to the ED. This study provides information on the process of care for older patients with COVID-19 in the ED, which can be useful to develop accurate diagnosis and treatment strategies when designing future protocols.

Comorbidities such as hypertension, obesity, diabetes, chronic cardiovascular and respiratory disease, cerebrovascular disease, dementia, kidney disease, immunosuppressive conditions, and cancer have been described as prognostic factors in COVID-19 [9] irrespectively of age [14]. Although these conditions were more frequent as age increased, age was also associated with poor in-hospital and 30-day outcomes after adjustment for sex, comorbidities, and severity, thereby strengthening the evidence that age is an independent prognostic factor in COVID-19 [9, 15]. However, other circumstances related to ageing such as the degree of frailty or malnutrition also likely facilitate a worse course of the disease. Immunosenescence may produce a state of vulnerability to infectious disease due to slow, incoordinate, and inefficient immune response to SARS-Cov-2 [8]. In addition, the physiological changes induced by ageing may favour delay in diagnosis and misdiagnosis [4, 7, 14, 16]. In fact, at arrival to the ED, the oldest groups more frequently had an atypical presentation, hypoxemia [17] and other abnormal radiological [18] and laboratory [15] findings associated with severe COVID-19.

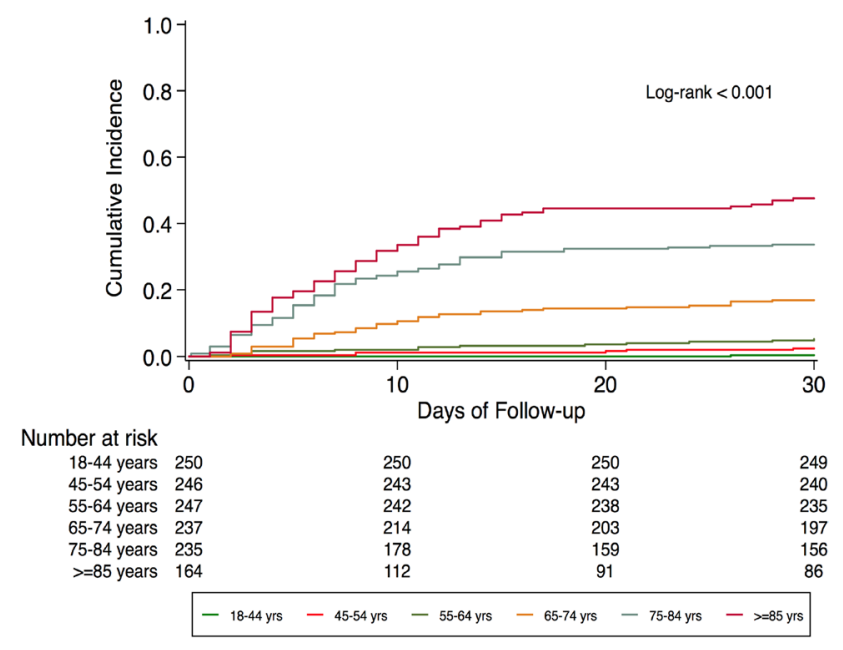

Fig. 2 Cumulative 30-day mortality curves for each age group
The clinical presentation on ED arrival differed between age groups. Subjective fever (80\%), cough (71\%), and dyspnoea $(47 \%)$ were the most common symptoms of presentation in our population similar to what has been described in the previous studies $[5,16,19-21]$. However, the presence of these core symptoms of COVID-19 was less common with increasing age. In addition, the older groups more frequently had unspecific findings such as asthenia or atypical presentations such as confusion and presyncope or syncope. This confirms the previous case reports which have described the importance of delirium and syncope (near-syncope and falls) as the first presentations in older patients with COVID-19 [22-24].

Regarding vital signs at arrival to the ED, the mean oxygen saturation and body temperature showed an inverse correlation with age. Although there was no relation between dyspnoea and age groups, hypoxemia was more common with increasing age. It is essential to take this circumstance into account, since a lack of subjective shortness of breath may delay the diagnosis of COVID-19, and hypoxemia is a recognized prognostic factor in COVID-19 [17]. With respect to body temperature, the previous studies have shown that older people are less likely to develop fever with an infectious disease [25]. This has also been reported in COVID-19 [17]. The lack of this cardinal sign may increase the difficulty in diagnosing COVID-19. Indeed, some authors have suggested that the usual temperature threshold for fever in older patients be modified [26].

Although no differences were found between the mean time from symptom onset to arrival at the ED, radiological findings and laboratory determinations related to the severity of COVID-19 were more common with increasing age. The previous studies have reported that older patients were more likely to suffer severe pneumonia [27-29], lymphopenia, and elevation of inflammatory markers in comparison with younger patients [4]. This state may be related to a worse host response against infection or a further diagnostic delay in older patients with COVID-19.

Considering that the diagnosis of COVID-19 is more complicated in older patients, emergency physicians need to use a combination of high clinical suspicion, radiological and laboratory tests, and RT-PCR results to allow early diagnosis, isolation, infection control measures, and provision of supportive care. Some clinical and laboratory abnormalities more frequent in older patients who died, and this should be considered in older aged patients due to the consequent worse prognosis. In our population, the frequency of the use of RT-PCR increased with age, as did the non-conclusive results. In this sense, a negative nasopharyngeal swab should not exclude COVID-19 infection if there is high clinical suspicion or an atypical presentation [30, 31].

Although there is no specific treatment for COVID-19, the use of hydroxychloroquine, antivirals, corticosteroids, 


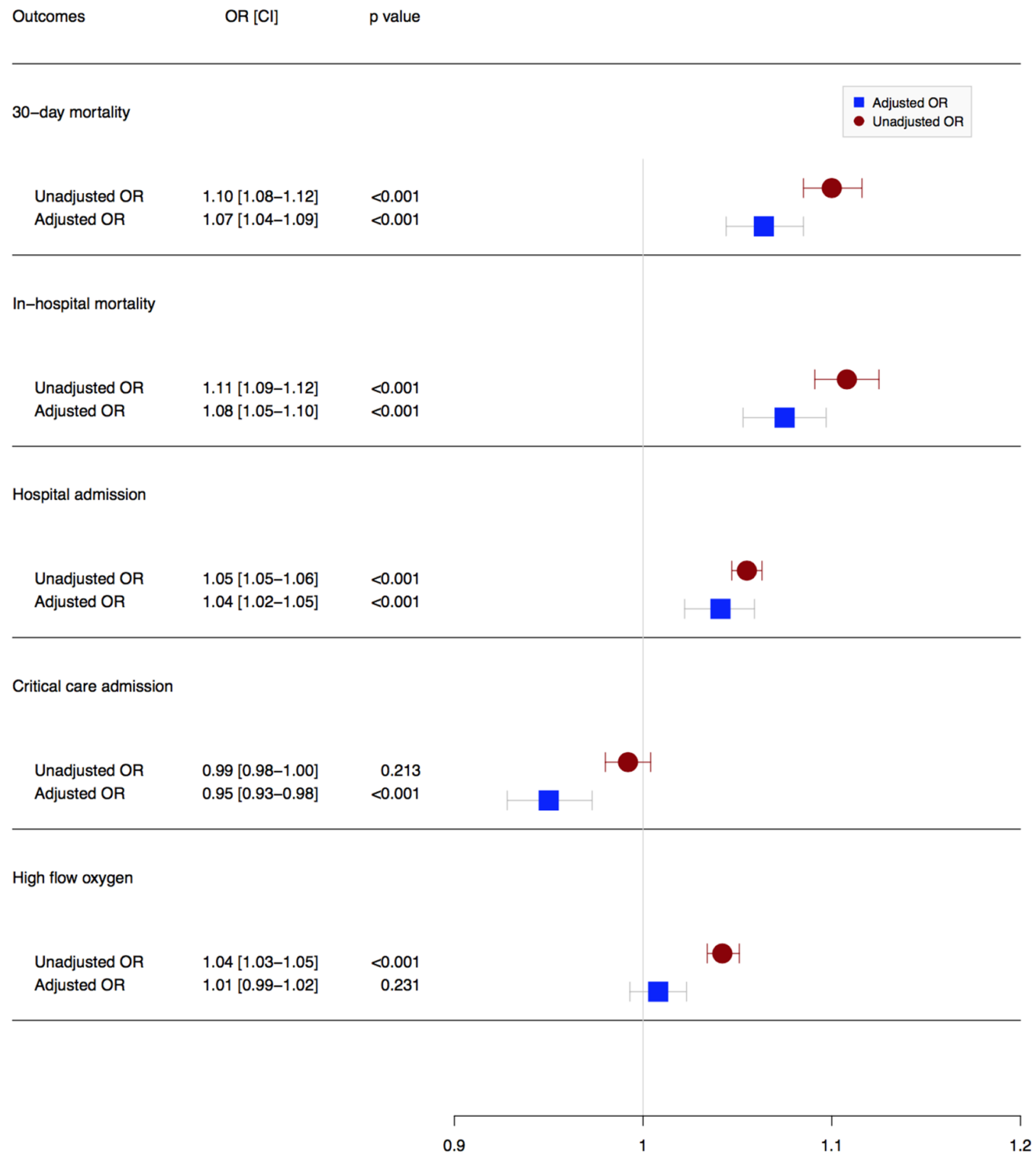

\footnotetext{
*Adjusted by sex, hypertension, diabetes, dyslipemia, obesity, chronic respiratory disease, heart disease, cerebrovascular disease, dementia, chronic renal disease, dependency on basic activities of daily living, oxygen saturation on arrival, abnormal chest x-ray, lymphocytes, platelet count, glomerular filtration rate, lactate dehydrogenase, D-Dimer, C-reactive protein and procalcitonin.
}

Fig. 3 Estimated unadjusted and adjusted Odds ratios for the association between age, as continues variables, and different outcomes

and antibiotics increased with age until 85 years old. After this age, the prescription of azithromycin and other antibiotics continued to increase but not the administration of hydroxychloroquine, lopinavir/ritonavir, or corticosteroids. This reduction may be related to the presence of more comorbidities and polypharmacy in these patients, which could favour drug interactions [32] as well as cautious use of corticosteroids to decrease the risk of adverse events or viral replication [33]. Regarding medication management in older adults during COVID-19, pharmacokinetics and drug interactions and medication reviews must be considered because of the increased risk of drug-related adverse events in older patients [33].

Patients should receive supportive care to help relieve symptoms, including care to support vital organ functions. In our study, age was a factor associated with not 
being admitted to intensive care, similar to what has been described in other series [4]. In this respect, it is striking that physicians have frequently faced complex decisions to determine who could most benefit from intensive care and ventilatory support in an overrun situation of health services and ICUs $[34,35]$. Prioritisation for ventilators or intensive care should not be based on chronological age. This age cut-off ignores the enormous heterogeneity of functional status, cognitive status, and burden of comorbidities in older populations [36]. Experts recommend a more holistic evaluation based on degree of frailty, the likelihood of medical interventions being successful, and the severity of the presenting acute illness to ensure the optimisation of available resources [37-39]. Decisions must be made on the basis of futility versus those based on rationing [40].

Our study has several limitations. First, this was a retrospective observational study and causal relationships between age and outcomes cannot be inferred. Second, the order of the diagnostic tests was determined by public health recommendations. In addition, the performance of RT-PCR in our centre may differ from the use of other diagnostic tests. These circumstances may have modified the categorisation of the patients and, therefore, this may have selected the population included in the study. Third, our study included information about patients who presented at the ED of one public hospital located in the centre of Madrid. This may introduce some degree of selection bias, since COVID-19 patients with mild symptoms could have been attended by EMS or GPs. The findings may be representative of what has happened in an urban and tertiary ED providing health care to one of the nuclei of the pandemic in Madrid. Fourth, this study was performed in an overcrowded ED during the COVID-19 pandemic. This may introduce some degree of information bias because of the high number of people involved in data collection. However, all the investigators involved in the collection of data had received the previous training and all the information was checked by a trained researcher. Fifth, this is a real-life cohort in which attending physicians followed the interventions of the local protocol. In addition, the recommendations on treatment for COVID-19 varied during the study period. Although this imposes limitations to our study, there is currently no evidence related to a specific drug, thereby making our findings more generalisable.

In conclusion, clinical presentation, management, and short-term outcomes of patients with COVID-19 admitted to the ED differs according to age-dependent categories. Age has an independent prognostic value and may help in shared decision-making. Further studies in older people with COVID-19 are needed to determine the factors associated with these poor outcomes.
Acknowledgements We wish to thank the Unidad de Innovación (UI); Unidad de Investigación Clínica y Ensayos Clínicos (UICEC); Unidad de Apoyo Metodológico a la Investigación (UAMI); Biobanco; Unidad de Apoyo a los Comités de Etica (UACE; Unidad de Apoyo a Farmacia: gestión de medicación de investigación (UFAR); and Laboratorios Gabinetes y Servicios Clínicos de Apoyo a la Investigación (LABSERCLI) for their most valuable effort.

Investigators of IdISSC-COVID-TASKFORCE and COVID-19 URG-HCSC Register: Carlos Javier Llamas, Laura Matilla, $M^{\mathrm{a}}$ Luisa Bretón, Beatriz Rojano, José Antonio Bustamante, Eric Jorge García Lamberechts, Manuel Maroto, Álvaro Martin Ruiz, María Martínez Agüero, Arturo Corbatón, Cesario Fernández Alonso, Jaime Abelaira, Pablo Matías, Raúl Perales, María Rosario Blázquez, M. Carmen Muñoz, Alejandra Ortega, Carlota Clemente, Sara Laínez, Antonio Trino Salto Ariza, Pedro Villarroel González Elipe, Juan Manuel Algarra, Javier Candel, Encarnación Fernández del Palacio, Rosa Moreno Rodríguez, Daniel Muñoz Jiménez, Vicente Estrada, María Jesús Téllez, Ángel Molino, María José Núñez, Noemí Cabello, Nieves Martel, Myriam Calle, Miguel Holagado María Luaces, Germán Seara, Joan Cháfer, Ángel Luis del Rey, Mercedes Martínez-Novillo, Alberto Delgado-Iribarren, $\mathrm{M}^{\mathrm{a}}$ Ángeles Cuadrado Cenzual, María José Torrejón Martínez, Fernando Ataúlfo González Fernández, Silvia Sánchez Ramón, Asunción Mora, Ana Arribi Vilella, Miguel Fernández Armero, José Manuel Martínez Sesmero, Rocío Manzano, Virginia Puebla, Juan Arrazola, Ana Bustos, Laura Galván, Ana Mañas, Angel Nava Muñoz, Sara Gómez Peña, Beatriz Cabeza Martínez, Irene Martín López, Joaquín López Herraiz, Alfonso Calle, Miguel Ángel Rubio Herrera, Isabel Runckle, Eduardo Anguita Mandley, Blanca García Reneses, David Fraguas, Marina Díaz Marsa, José Luis Carrasco, Julia Sevilla, Iabel Ramos, Marta Navas, Aránzazu Álvarez de Arcaya, José Ramón Núñez, Mar García Arenillas, Lourdes Cabrera, Carlos Macaya, Fernando Macaya, Iván Nuñez-Gil, Jorge Matías-Guiu, Jesús Porta, Miguel Sánchez, Fernando Martínez Sagasti, Leonor Laredo, Emilio Vargas, Alberto Mariano Lázaro, Carmen de Burgos, Manuel Fuentes Ferrer, Gloria Mato Chian, Beatriz Peláez Ros, María Ángeles Valcárcel de la Iglesia, Náyade del Prado, José M ${ }^{a}$ Leal Pozuelo, José Luis Fernández Rueda, Víctor Hernández Martín-Romo,Elena Urcelay, Antonio Portolés, and Julio Mayol.

Author contributions FJMS conceived the study and conducted the analysis. FJMS and AVC supervised the reporting of the Registry and data collection. FJMS and CFP provided statistical advice on study design and analysed the data. The remaining co-authors undertook patient recruitment. FJMS, ET, and EC drafted the manuscript, although all authors contributed substantially to its revision, particularly PLA, FCT, CMV, JGC, and ALP. FJMS takes responsibility for the paper as a whole.

Funding This Research Group has not received any funding.

\section{Compliance with ethical standards}

Conflict of interest The authors have nothing to disclose in relationship with this manuscript.

Ethical approval The COVID-19-URG-HCSC registry was developed following the ethical principles of the Declaration of Helsinki for medical research in humans. The protocol was approved by the HCSC Research Ethics Committee (Cl 20/338-E).

Informed consent The waiver of the need for obtaining informed consent was approved by the HCSC Research Ethics Committee $(\mathrm{Cl}$ 20/338-E). 


\section{References}

1. World Health Organization (2020) Coronavirus disease (COVID19) outbreak. Second meeting of the Emergency Committee meeting. https://www.who.int. Accessed 17 May 2020

2. Equipo COVID-19 (2020) Red Nacional de Vigilancia Epidemiologica-Instituto Carlos III. Informe no 29. Situación de COVID-19 en España a 14 de mayo de 2020. https://www.isciii.es/QueHa cemos/Servicios/VigilanciaSaludPublicaRENAVE/Enfermedad esTransmisibles/Documents/INFORMES/Informes\%2520C OVID-19/Informe\%2520n\%C2\%BA\%252031.\%2520Situac i $\%$ C3\% B 3n \% 2520de\%2520COVID-19\%2520en $\% 2520 \mathrm{E}$ spa\%C3\%B1a\%2520a\%252014\%2520de\%2520mayo\%2520d e\%25202020.pdf. Accessed 17 May 2020

3. Pérez Díaz J, Abellán García A, Aceituno Nieto P, Ramiro Fariñas D (2020) Un perfil de las personas mayores en España, 2020. Indicadores estadísticos básicos. https://envejecimiento.csic.es/docum entos/documentos/enred-indicadoresbasicos2020.pdf. Accessed 17 May 2020

4. Liu K, Chen Y, Lin R, Han K (2020) Clinical features of COVID19 in elderly patients: a comparison with young and middle-aged patients. J Infect 80:e14-e18

5. Guan WJ, Ni ZY, Zhong NS et al (2020) Clinical characteristics of 2019 novel coronavirus infection in China. N Engl J Med 382:1708-1720

6. Cope DG (2013) Polypharmacy in older adults: the role of the advanced practitioner in oncology. J Adv Pract Oncol 4:107-112

7. Chen T, Dai Z, Mo P, Li X, Ma Z, Song S et al (2020) Clinical characteristics and outcomes of older patients with coronavirus disease 2019 (COVID-2019) in Wuhan, China: a single-centered, retrospective study. J Gerontol A Biol Sci Med Sci. https://doi. org/10.1093/gerona/glaa089

8. Nikolich-Zugich J, Knox KS, Rios CT, Natt B, Bhattacharya D, Fain MJ (2020) SARS-CoV-2 and COVID-19 in older adults: what we may expect regarding pathogenesis, immune responses, and outcomes. Geroscience 42:505-514

9. Williamson E, Walker AJ, Bhaskaran KJ, Bacon S, Bates C, Morton CE et al (2020) OpenSAFELY: factors associated with COVID-19-related hospital death in the linked electronic health records of 17 million adult NHS patients. Cold Spring Harb Lab. https://doi.org/10.1101/2020.05.06.20092999

10. Lloyd-Sherlock PG, Kalache A, McKee M, Derbyshire J, Geffen L, Casas FG (2020) WHO must prioritise the needs of older people in its response to the covid-19 pandemic. BMJ 368:1164

11. Instituto de Salud Carlos III (2020) Procedimiento de actuación frente a casos de nuevo coronavirus. Actualización 31.03.2020. https://bvsalud.isciii.es/lis-profesionales/lis-search/resou $\mathrm{rce} / \mathrm{id}=46956$. Accessed 10 May 2020

12. WHO/ISARIC. Clinical Data Collection-The COVID-19 Case Report Forms (CRFs) [Internet]. https://isaric.tghn.org/covid-19clinical-research-resources/. Accessed 10 May 2020

13. Instituto de Salud Carlos III (2020) Manejo en urgencias de pacientes con sospecha de COVID-19. Versión de 9 de marzo de febrero de 2020. https://www.mscbs.gob.es/profesionales/salud Publica/ccayes/alertasActual/nCov-China/documentos/Manej o_urgencias_pacientes_con_COVID-19.pdf. Accessed 10 May 2020

14. Wang L, He W, Yu X, Hu D, Bao M, Liu H et al (2020) Coronavirus disease 2019 in elderly patients: characteristics and prognostic factors based on 4-week follow-up. J Infect 80:639-645

15. Sun H, Ning R, Tao Y, Yu C, Deng X, Zhao C et al (2020) Risk factors for mortality in 244 older adults with COVID-19 in Wuhan, China: a retrospective study. J Am Geriatr Soc. https:// doi.org/10.1111/jgs.16533
16. Niu S, Tian S, Lou J, Kang X, Zhang L, Lian H et al (2020) Clinical characteristics of older patients infected with COVID-19: a descriptive study. Arch Gerontol Geriatr 89:104058

17. Xie J, Covassin N, Fan Z, Singh P, Gao W, Li G, Kara T, Somers VK (2020) Association between hypoxemia and mortality in patients with COVID-19. Mayo Clin Proc 95(6):1138-1147. https ://doi.org/10.1016/j.mayocp.2020.04.006

18. Yu Q, Wang Y, Huang S, Liu S, Zhou Z, Zhang S et al (2020) Multicenter cohort study demonstrates more consolidation in upper lungs on initial CT increases the risk of adverse clinical outcome in COVID-19 patients. Theranostics 10:5641-5648

19. Huang C, Wang Y, Li X, Ren L, Zhao J, Hu Y et al (2020) Clinical features of patients infected with 2019 novel coronavirus in Wuhan, China. Lancet 395(10223):497-506

20. Richardson S, Hirsch JS, Narasimhan M, Crawford JM, McGinn T, Davidson KW et al (2020) Presenting characteristics, comorbidities, and outcomes among 5700 patients hospitalized with COVID-19 in the New York City Area. JAMA. https://doi. org/10.1001/jama.2020.6775

21. Gold JA, Wong KK, Szablewski CM et al (2020) Characteristics and clinical outcomes of adult patients hospitalized with COVID19-Georgia, March 2020. Morb Mortal Wkly Rep 69:545-550

22. Alkeridy WA, Almaglouth I, Alrashed R, Alayed K, Binkhamis $\mathrm{K}$, Alsharidi A et al (2020) A unique presentation of delirium in a patient with otherwise asymptomatic COVID-19. J Am Geriatr Soc. https://doi.org/10.1111/jgs.16536

23. O'Hanlon S, Inouye SK (2020) Delirium: a missing piece in the COVID-19 pandemic puzzle. Age Ageing

24. Chen T, Hanna J, Walsh EE, Falsey AR, Laguio-Vila M, Lesho E (2020) Syncope, near-syncope, or non-mechanical falls as a presenting feature of COVID-19. Ann Emerg Med. https://doi. org/10.1016/j.annemergmed.2020.04.037

25. Norman DC (2000) Fever in the elderly. Clin Infect Dis $31: 148-151$

26. Leung C (2020) Risk factors for predicting mortality in elderly patients with COVID-19: a review for clinical data in China. Mech Ageing Dev 188:111255

27. Cao Y, Liu X, Xiong L, Cai K (2020) Imaging and clinical features of patients with 2019 novel coronavirus SARS-CoV-2: a systematic review and meta-analysis. J Med Virol. https://doi. org/10.1002/jmv.25822

28. Chen Z, Fan H, Cai J, Li Y, Wu B, Hou Y (2020) High-resolution computed tomography manifestations of COVID-19 infections in patients of different ages. Eur J Radiol 126:108972

29. Zhu T, Wang Y, Zhou S, Zhang N, Xia L (2020) A comparative study of chest computed tomography features in young and older adults with corona virus disease (COVID-19). J Thorac Imaging. https://doi.org/10.1097/RTI.0000000000000513

30. Isaia G, Marinello R, Tibaldi V, Tamone C, Bo M (2020) Atypical presentation of Covid-19 in an older adult with severe alzheimer disease. Am J Geriatr Psychiatry

31. Yang DM, Chang TJ, Wang ML, Tsai PH, Lin TH, Wang CT et al (2020) Hunting coronavirus SARS-CoV-2 (2019-nCoV): from laboratory testing back to basic research. J Chin Med Assoc. https ://doi.org/10.1097/JCMA.0000000000000332

32. Back D, Marzolini C, Hodge C, Marra F, Boyle A, Gibbons S, et al (2020) COVID-19 treatment in patients with comorbidities: Awareness of drug-drug interactions. Br J Clin Pharmacol

33. Ailabouni NJ, Hilmer SN, Kalisch L, Braund R, Reeve E (2020) COVID-19 pandemic: considerations for safe medication use in older adults with multimorbidity and polypharmacy. J Gerontol A Biol Sci Med Sci 
34. Falvey JR, Ferrante LE (2019) Frailty assessment in the ICU: translation to 'real-world' clinical practice. Anaesthesia 74:700-703

35. Saxena A, Horby P, Amuasi J, Aagaard N, Köhler J, Gooshki ES et al (2019) Ethics preparedness: facilitating ethics review during outbreaks-recommendations from an expert panel. BMC Med Ethics 20:29

36. Farrell TW, Francis L, Brown T, Ferrante LE, Widera E, Rhodes R et al (2020) Rationing limited health care resources in the COVID-19 era and beyond: ethical considerations regarding older adults. J Am Geriatr Soc. https://doi.org/10.1111/jgs.16539

37. Rubio O, Estella A, Cabre L, Saralegui-Reta I, Martin MC, Zapata L et al (2020) Ethical recommendations for a difficult decisionmaking in intensive care units due to the exceptional situation of crisis by the COVID-19 pandemia: a rapid review \& consensus of experts. Med Intensiva
38. Chong E, Chan M, Tan HN, Lim WS (2020) COVID-19: use of the clinical frailty scale for critical care decisions. J Am Geriatr Soc. https://doi.org/10.1111/jgs.16528

39. Hubbard RE, Maier AB, Hilmer SN, Naganathan V, Etherton-Beer C, Rockwood K (2020) Frailty in the Face of COVID-19. Age Ageing

40. Le Couteur DG, Anderson RM, Newman AB (2020) COVID-19 is a disease of older people. J Gerontol A Biol Sci Med Sci

Publisher's Note Springer Nature remains neutral with regard to jurisdictional claims in published maps and institutional affiliations.

\section{Affiliations}

\section{F. Javier Martín-Sánchez ${ }^{1,2,3}$ (1) - Enrique del Toro ${ }^{1} \cdot$ Eduardo Cardassay $^{1} \cdot$ Adrián Valls Carbó $^{4} \cdot$ Federico Cuesta $^{5}$. Marta Vigara ${ }^{5}$. Pedro Gil ${ }^{5}$. Amanda López López Picado ${ }^{3}$. Carmen Martínez Valero ${ }^{3}$. Juande D. Miranda ${ }^{6}$. Pedro Lopez-Ayala ${ }^{7}$. David Chaparro ${ }^{1}$. Gabriel Cozar López ${ }^{1} \cdot$ María del Mar Suárez-Cadenas ${ }^{1,3}$. Pablo Jerez Fernández ${ }^{1}$ - Beatriz Angós ${ }^{1}$. Cristina Díaz del Arco $^{8}$. Esther Rodríguez Adrada ${ }^{3}$. María Teresa Montalvo Moraleda ${ }^{4} \cdot$ Carolina Espejo Paeres $^{9} \cdot$ Cesáreo Fernández Alonso $^{1,3} \cdot$ Carlos Elvira $^{10}$. Ana Chacón ${ }^{10}$. Miguel Ángel García Briñón ${ }^{1,3}$. José Luis Fernández Rueda ${ }^{11}$. Luis Ortega ${ }^{8}$. Cristina Fernández Pérez ${ }^{12}$. Juan Jorge González Armengol ${ }^{1,2,3}$. Juan González del Castillo ${ }^{1,3}$}

F. Javier Martín-Sánchez fjjms@hotmail.com

1 Department of Emergency, Hospital Clínico San Carlos, Calle Profesor Martín-Lagos s/n, 28040 Madrid, Spain

2 Faculty of de Medicine, Universidad Complutense de Madrid, Madrid, Spain

3 Institute for Health Research of Hospital San Carlos, Madrid, Spain

4 Department of Neurology, Hospital Clínico San Carlos, Madrid, Spain

5 Department of Geriatric, Hospital Clínico San Carlos, Madrid, Spain

6 Risk Modelling Division of Repsol, Madrid, Spain
7 Cardiovascular Research Institute Basel (CRIB), Department of Cardiology, University Hospital Basel, University of Basel, Basel, Switzerland

8 Department of Pathological Anatomy, Hospital Clínico San Carlos, Madrid, Spain

9 Department of Cardiology, Hospital Clínico San Carlos, Madrid, Spain

10 Department of Admissions and Clinical Documentation, Hospital Clínico San Carlos, Madrid, Spain

11 Department of Information Systems and Technologies, Hospital Clínico San Carlos, Madrid, Spain

12 Department of Preventive Medicine, Hospital Clínico San Carlos, Madrid, Spain 